(e-migrinter

e-Migrinter

9| 2012

Immigrés, illégaux, réfugiés. Questions sur les

enquêtes et les catégories

\title{
Enquêter auprès des réfugiés libériens dispersés à Conakry : approche par les réseaux sociaux
}

Hélène Simon-Lorière

\section{CpenEdition}

\section{Journals}

Édition électronique

URL : https://journals.openedition.org/e-migrinter/768

DOI : 10.4000/e-migrinter.768

ISSN : 1961-9685

Éditeur

UMR 7301 - Migrinter

Édition imprimée

Date de publication : 20 juillet 2012

Pagination : 47-63

ISSN : 1961-9685

Référence électronique

Hélène Simon-Lorière, "Enquêter auprès des réfugiés libériens dispersés à Conakry : approche par les réseaux sociaux », e-Migrinter [En ligne], 9 | 2012, mis en ligne le, consulté le 20 mai 2021. URL : http:// journals.openedition.org/e-migrinter/768; DOI : https://doi.org/10.4000/e-migrinter.768 


\section{Enquêter auprès des réfugiés libériens dispersés à Conakry : approche par les réseaux sociaux}

Hélène Simon-Lorière

\begin{abstract}
$\mathbf{L}$
es difficultés méthodologiques que rencontrent les chercheurs sur leurs terrains font l'objet de réflexion et d'échanges en géographie ${ }^{1}$. Terme porteur de représentations diverses, le terrain renvoie à l'espace étudié et au lieu où se déploie une méthode (Calbérac, 2010, pp. 347-355). Cet article se propose de présenter et de discuter certains éléments de la politique du terrain élaborée pour un travail de thèse auprès de réfugiés libériens à Conakry en Guinée.
\end{abstract}

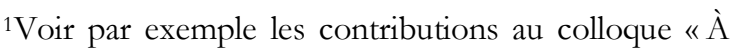
travers l'espace de la méthode : les dimensions du terrain en géographie » qui s'est tenu à Arras en juin 2008: $\quad$ http://terrain.ens-lyon.fr/programme13160.kjsp?RH=TERRAIN010000

\section{Introduction}

Cet article se propose de présenter et de discuter certains éléments de la «politique du terrain » (Olivier de Sardan, 1995) que j'ai élaborée pour mon travail de thèse auprès des réfugiés libériens à Conakry en Guinée. Ce terrain d'enquête est à bien des égards un «terrain sensible», selon la définition que proposent les anthropologues Florence Bouillon, Marion Fresia et Virginie Tallio (2006, pp. 13-15) : «Nous donnons ici un triple sens au qualificatif sensible. En premier lieu, les terrains sont sensibles en ce qu'ils sont porteurs d'une souffrance sociale, d'injustice, de domination, de violence. (...) En second lieu, ils sont sensibles parce qu'ils impliquent de renoncer à un protocole d'enquête par trop canonique (...). En dernier lieu, ces terrains s'avèrent sensibles en ce qu'ils relèvent d'enjeux sociopolitiques cruciaux ». Les réfugiés rencontrés à Conakry ont en effet connu des expériences douloureuses de guerre et d'exil, ce qui correspond au premier élément mis en avant par ces auteures. Les enjeux géopolitiques et sociopolitiques de leur présence en Guinée sont également complexes et justifient d'employer le qualificatif sensible au sens où les réfugiés ont été stigmatisés au début des années 2000 comme des «rebelles » apportant la guerre en Guinée (McGovern, 2002). Mais c'est surtout l'aspect méthodologique qui sera développé ici pour montrer les difficultés rencontrées sur le terrain et la façon dont j'ai essayé de les contourner, si ce n'est de les surmonter, en recourant aux réseaux sociaux.

La Guinée est le pays voisin du Liberia qui a reçu, d'après le Haut Commissariat des Nations Unies pour les réfugiés (HCR), le plus grand nombre de réfugiés pendant les 
deux guerres civiles libériennes entre 1990 et 2003 avec plus d'un demi-million de réfugiés libériens sur son territoire (voir figure $\mathrm{n}^{\circ} 1$ ). La majorité des réfugiés est restée dans des camps en Guinée forestière, région proche du Liberia, où plusieurs recherches ont été menées (voir par exemple Black, 1999 ; Van Damme, 1999; Ferry, 2004; Andrews, 2008 ; Agier, 2008).

Figure $\mathbf{n}^{\circ} \mathbf{1}$ : Les réfugiés libériens accueillis en Afrique de l’Ouest en 1996.

\begin{tabular}{|l|c|}
\hline $\begin{array}{l}\text { Pays d'asile en } \\
\text { Afrique de } \\
\text { l'Ouest }\end{array}$ & $\begin{array}{l}\text { Réfugiés libériens recensés } \\
\text { par le HCR en 1996 }\end{array}$ \\
\hline Guinée & 663854 \\
\hline Côte d'Ivoire & 327288 \\
\hline Sierra Leone & 13521 \\
\hline Ghana & 15178 \\
\hline Nigéria & 5278 \\
\hline
\end{tabular}

Source: UNHCR Statistical yearbooks

NB : Le Liberia comptait 2,1 millions d'babitants en 1984 (dernier recensement national avant la guerre civile).

La particularité de mon travail de thèse est d'être concentrée sur les réfugiés libériens installés dans la capitale guinéenne, Conakry, en dehors de ce système de camps ou l'ayant quitté. Les réfugiés urbains sont un sujet de recherche récent, pour le HCR comme pour les chercheurs qui travaillent sur les migrations forcées (Jacobsen, 2006). Mon étude s'inscrit dans ce mouvement en apportant un regard géographique dans un champ, essentiellement anglophone, dominé par les juristes et les anthropologues. Il s'agit d'un terrain de recherche nouveau, qui présente une particularité géographique par rapport à la concentration spatiale des camps de réfugiés près des frontières : la dispersion des réfugiés en ville. En effet, à la différence d'autres villes (Accra au Ghana par exemple), il n'y a pas de camp de réfugiés urbains à Conakry si bien que les réfugiés libériens à Conakry vivent au milieu de la population guinéenne ${ }^{2}$. Leur présence en ville est un sujet moins sensible que dans d'autres grandes villes africaines, comme à Dar Es Salam en Tanzanie où les réfugiés

${ }^{2}$ Dans mon travail de thèse, j'interroge d'ailleurs les conséquences de cette dispersion géographique pour les conditions de vie des réfugiés, leur relation avec les locaux et leurs projets migratoires, en comparant la situation des réfugiés libériens à Conakry avec celle de leurs compatriotes à Accra, qui vivent concentrés dans le camp de Buduburam. sont soumis à un permis de résidence délivré par les autorités (Willems, 2003, p. 89). Le gouvernement guinéen n'a jamais interdit aux réfugiés de venir en ville mais l'aide humanitaire a été réservée aux réfugiés en Guinée forestière dès 1990 (Van Damme, 1999 , p. 353) et la liberté d'installation des réfugiés dans la capitale comme dans tout le pays a été contestée dans les années 19992001 en lien avec les attaques rebelles aux frontières de la Guinée (Mc Govern, 2002).

L'objectif de mon travail de thèse était d'essayer de saisir les projets migratoires des réfugiés libériens installés à Conakry, au moment même où la situation qui avait causé leur exil prenait fin ${ }^{3}$. La fin des années 2000 correspond en effet pour les Libériens au retour de la paix dans leur pays : en 2006, trois ans après la fin de la dernière guerre civile et le départ de Charles Taylor ${ }^{4}$, Ellen Johnson Sirleaf a été élue présidente du Liberia. À partir de 2004, le HCR a

${ }^{3}$ Mes recherches à Conakry auprès des réfugiés libériens se sont étalées entre 2007 et 2009 et les différents séjours mis bout à bout représentent un an de terrain en Guinée.

${ }^{4}$ Charles Taylor a été le principal chef de guerre de la première guerre civile libérienne (1989-1996) et a été élu président en 1997 avant de voir son pouvoir contesté par de nouveaux mouvements rebelles provoquant la deuxième guerre civile libérienne (1999-2003). 
encouragé le retour au pays des Libériens en exil suivant le présupposé que le «rapatriement est la meilleure solution durable pour les réfugiés $»^{5}$ et en mettant en place des programmes d'accompagnement au retour jusqu'en 2007 mais qui ont surtout eu du succès en Guinée parmi les réfugiés des camps. Une des questions à l'origine de ma thèse était de comprendre pourquoi certains réfugiés restaient en arrière quand la majorité de leurs compatriotes rentraient, et pourquoi ces réfugiés se trouvaient surtout en ville, ici dans la capitale guinéenne. Il s'agissait d'explorer les possibilités offertes à ces réfugiés pour essayer de comprendre leur hésitation à rentrer et de tenter de percevoir la façon dont les réfugiés, ayant subi un déplacement forcé, s'efforcent de se réapproprier leur choix d'un lieu de vie. D’après ce que j’ai observé, les réfugiés urbains qui envisagent de rentrer prennent leur temps et font des allers et retours réguliers entre Conakry et Monrovia pour évaluer la situation au Liberia. Cependant, la plupart des Libériens de Conakry restent dans la ville car ils espèrent pouvoir partir vers les États-Unis ou un autre pays développé grâce aux programmes d'accueil de réfugiés africains. Pourtant, leur attente est souvent vaine car rares sont ceux qui obtiennent de partir de Conakry et d'être ainsi « réinstallés », selon l'expression du HCR. Enfin, les évènements politiques de la fin du «règne» de Lansana Conté, le président de la Guinée de 1984 à 2009, ont inquiété les réfugiés et entretenu l'incertitude sur leur séjour en Guinée. Face à ce questionnement sur les projets migratoires, l'outil du réseau social était un moyen de percevoir les possibles points d'ancrages de ces réfugiés libériens, en Guinée, au Liberia, mais aussi dans d'autres pays de la sousrégion, voire du monde.

Le terme de réseau social est ici à comprendre comme les relations des réfugiés avec d'autres individus, qu'ils soient

${ }^{5} \mathrm{On}$ peut le lire notamment sur le site internet du HCR : http://www.unhcr.be/fr/profil/qui-nousaidons/rapatries.html (Consulté le 13 avril 2012). libériens, guinéens ou d'autres nationalités, et qu'ils vivent à Conakry, en Guinée, au Liberia ou ailleurs dans le monde. Pierre Mercklé définit en effet le réseau social comme l'ensemble des relations entre individus (Mercklé, 2004, p. 11). Les réseaux sociaux ont été un outil pour le recueil de données empiriques autour du projet migratoire lui-même, une façon d'aborder de biais les possibilités migratoires existantes pour les individus rencontrés. Mais ils ont d'abord et surtout été un outil pour pouvoir mener des enquêtes auprès des réfugiés libériens à Conakry, pour accéder au terrain en quelque sorte. Je me suis intéressée tout particulièrement aux relations des réfugiés libériens avec d'autres réfugiés libériens afin de les trouver dans la capitale guinéenne où ils sont peu nombreux et dispersés.

D’une part, il s'agit de présenter ici la façon dont cet outil des réseaux sociaux a été mobilisé concrètement et localement pour répondre aux contraintes d'un terrain sensible, c'est-à-dire à la situation de ces réfugiés libériens à Conakry. D'autre part, il s'agit d'utiliser ces réseaux comme un élément d'étude à part entière pour appréhender les migrations de fuite tous azimuts causées par la guerre du Liberia et leur éventuelles conséquences en termes de projets migratoires pour les Libériens à Conakry. Dans un premier temps, je présenterai les difficultés rencontrées pour identifier les réfugiés libériens à Conakry et les façons d'accéder au terrain que j'ai écartées avant de privilégier l'approche par les réseaux sociaux : recours à des données statistiques, identification de quartiers d'études et appui sur le HCR ou sur des organisations humanitaires engagées auprès des réfugiés. Ensuite, j’expliquerai comment j'ai mis en œuvre cette approche avec la méthode dite de « la boule de neige » sans en ignorer les possibles limites, mais en montrant aussi son rôle pour gagner la confiance des enquêtés. Enfin en conclusion, je reviendrai sur ce que cette méthode m'a apporté sur ce terrain sensible, au-delà d'un accès aux individus à enquêter, 
en évoquant quelques éléments de résultats sur les perspectives migratoires découvertes par la reconstitution des réseaux sociaux des réfugiés libériens de Conakry.

\section{Chercher les réfugiés}

Pour présenter la «politique de terrain » que j'ai adoptée, c'est-à-dire atteindre une plausibilité et une validité pour ce travail (Olivier de Sardan, 1995), je propose d'abord d'évoquer certains modes d'accès au terrain que j'ai explorés avant de les écarter: celui de l'appui sur des statistiques, celui du choix de quartiers d'études et celui de l'intermédiaire du HCR pour rencontrer des réfugiés. Chacun de ces procédés s'est avéré problématique pour des raisons concrètes mais aussi parce qu'ils ne convenaient pas pour essayer de répondre à mes questionnements sur les projets migratoires des réfugiés.

\section{Avoir une vision statistique globale? Mission impossible}

L'une de mes premières démarches en Guinée a été d'essayer de connaittre le nombre de réfugiés libériens présents dans la capitale. Or l'essentiel des efforts statistiques concernant les réfugiés s'est concentré sur la Guinée forestière, lieu principal d'accueil des réfugiés. Le recensement de la population guinéenne de 1996 le met particulièrement en avant (Bidou, Touré, 2002), d'autant plus que cette année correspond à un pic dans l'accueil des réfugiés du Liberia et de la Sierra-Léone voisines en guerre. Les villes frontalières figurent parmi celles qui ont enregistré la plus forte croissance, en s'appuyant sur l'afflux des réfugiés, et JeanEtienne Bidou ainsi que Julien Gbéré Toure soulignent que les réfugiés ont ainsi participé au renforcement des centres urbains secondaires dans les années 1990. À l'exception d'un recensement des réfugiés mené en 1991 par l'Institut national de la statistique dans cette seule région de Guinée forestière, les services de l'État guinéen n'ont pas tenu de statistiques spécifiques sur les réfugiés en général ni sur leur présence à Conakry. Ils se sont plutôt appuyés sur celles produites par les organisations humanitaires, notamment celles du HCR. Quand je me suis rendue dans les mairies, les ministères ou le Bureau national des réfugiés, on m'a renvoyée à chaque fois vers le HCR, considéré comme le véritable responsable des réfugiés. Ce dernier produit effectivement des statistiques, à travers des statistical yearbooks publiés en ligne pour les années $2000^{6}$, à l'échelle nationale et pour les lieux d'accueil des réfugiés en Guinée, c'està-dire surtout les camps de réfugiés. Dans les camps, il est nécessaire de compter les réfugiés pour leur distribuer l'aide alimentaire ou faciliter leur accès à des soins médicaux, même si ces recensements ne se font pas sans difficultés (Crisp, 1999). À Conakry, le nombre de réfugiés était nettement moins important mais aussi moins bien connu.

N'ayant pas droit à une aide alimentaire, ils n'étaient pas régulièrement recensés et ils ne vivaient pas dans une zone désignée par les autorités guinéennes ou le HCR mais dispersés dans la ville. Cependant, après les attaques menées dans la région frontalière en 2000 et les vagues d'arrestations de réfugiés à Conakry, le HCR a cherché à mieux connaittre ces réfugiés urbains. Le statistical yearbook de 2005 annonce ainsi 11320 réfugiés enregistrés par le HCR à Conakry, mais sans détailler leur nationalité. En m'adressant au bureau du HCR à Conakry, j'ai obtenu des chiffres plus précis : le HCR enregistrait 4054 réfugiés libériens (sur un total de 8944 réfugiés) début $2008^{7}$. Un employé du HCR a accepté de me donner un fichier statistique avec quelques informations sur les profils des réfugiés libériens ${ }^{8}$. Cette base de données

${ }^{6}$ Voir :

http://www.unhcr.org/cgi$\mathrm{bin} / \mathrm{texis} / \mathrm{vtx} / \mathrm{search} ? \mathrm{page}=49 \mathrm{e} 484 \mathrm{c} 66 \& \mathrm{coi}=\mathrm{GIN} \& \mathrm{c}$ id=49aea93aba.

${ }^{7} \mathrm{La}$ diminution du nombre total de réfugiés entre 2005 et 2008 s'explique par les départs de SierraLéonais, et dans une moindre mesure de Libériens, dans le cadre des programmes de rapatriement.

${ }^{8}$ Entretien avec un employé du HCR chargé du suivi 
n'est pas devenue un point de départ pour mon travail mais a pu constituer une référence, notamment démographique et socio-économique. Le tableau apporte en effet des informations sur le sexe et la classe d'âge des individus, informations enregistrées automatiquement lors de la délivrance de l'attestation de réfugié. Le HCR ayant cherché à mettre en place des programmes de micro-crédits ou de formation pour améliorer les conditions de vie des réfugiés, le tableau indique l'activité professionnelle pour la plupart des individus selon de grandes catégories. Néanmoins, il est peu exploitable d'un point de vue géographique car il ne renseigne pas le lieu d'origine des individus au Liberia, mais seulement leur ethnie déclarée, et ce de façon lacunaire. Il ne comporte pas d'information sur les parcours de migration forcée des Libériens, alors même que de nombreux réfugiés installés à Conakry étaient souvent passés par des camps gérés par le HCR en Guinée ou dans d'autres pays. Un petit nombre a notamment rejoint la capitale guinéenne après le retrait de l'aide humanitaire et la fermeture officielle des camps de Guinée forestière pour les Libériens en $2007^{9}$. Enfin, il ne mentionne pas les lieux de résidence des réfugiés à Conakry. Cette information n'a pas été relevée de façon systématique auprès des réfugiés.

Ces statistiques du HCR correspondent au nombre de Libériens ayant

statistique, 6 février 2008.

${ }^{9}$ Lacey Andrews Gale a mené des enquêtes dans le camp de réfugiés sierra-léonais de Boréah en Guinée forestière entre 2001 et 2003 puis a gardé des contacts avec ses informateurs qui lui ont expliqué que certains réfugiés étaient restés dans les maisons qu'ils s'étaient construites, même après le retrait des organisations humanitaires en 2006. Elle souligne le paradoxe de ces camps "fermés» par le HCR où pourtant une partie des habitants est restée, et pose la question de l'avenir de ces réfugiés et de leur éventuelle intégration en Guinée (Andrews, 2008). De la même façon, d'après mes observations lors d'un séjour en Guinée forestière en 2008, certains Libériens sont restés et continuent de vivre dans la zone qui constituait le camp de Kouankan près de Macenta. le statut juridique de réfugié et n'incluent donc pas ceux qui n'ont pas obtenu ou demandé cette reconnaissance institutionnelle de leur exil en Guinée. Pour mon travail de thèse, pour interroger les projets migratoires individuels, il m'a paru plus intéressant de choisir une définition plus large du terme « réfugié » et d'inclure toutes les personnes ayant fui le Liberia à cause de la guerre et résidant à Conakry. Pour croiser les sources, je me suis donc adressée à l'ambassade du Liberia à Conakry. Le chargé d'affaires, en poste à Conakry depuis 1989, a dit tenir un registre de ses ressortissants et avancé le nombre de 3500 Libériens à Conakry. Tous ces Libériens ne sont pas des réfugiés mais le diplomate a insisté sur le fait que de nombreuses personnes fuyant la guerre s'étaient adressées à l'ambassade pour obtenir des documents officiels, un hébergement, une aide matérielle. Certaines de ces personnes étaient enregistrées par le HCR, et avaient une "attestation de réfugié », d'autres non ${ }^{10}$. Il existe en effet des réfugiés statutaires, connus du HCR et figurant dans les statistiques de l'institution, et des réfugiés sans statut juridique. Certains Libériens ont bénéficié de l'accueil de leur famille élargie en Guinée forestière (Van Damme, 1999) et n'ont jamais demandé ce statut: à leur venue à Conakry, ils ont continué à vivre parmi la population guinéenne sans solliciter le HCR. D'autres ont cherché à obtenir des papiers de réfugiés mais l'attribution du statut est devenue plus stricte après les attaques de 2000: ils ont parfois été déboutés de leur demande par la commission du bureau guinéen des réfugiés instituée légalement au début des années 2000. Ayant conscience de la présence de ces

\footnotetext{
${ }^{10}$ Pour ces réfugiés statutaires, il y a là une contradiction juridique car ils sont déclarés avoir perdu la protection de leur État d'origine et ne sont pas censés s'adresser à leur ambassade à l'étranger. Le diplomate libérien justifie cette exception en s'appuyant sur la différence de traitement entre les réfugiés des camps et les réfugiés urbains et souligne que l'action de l'ambassade a aussi concerné des rapatriements vers le Liberia (Entretien avec le chargé d'affaires de l'ambassade du Liberia à Conakry, 21 février 2008).
} 
réfugiés sans papiers, les autorités guinéennes et le HCR ont organisé un recensement des réfugiés (libériens mais aussi sierra-léonais et ivoiriens) à Conakry fin 2007. Cette opération a été menée pour mieux connaittre les réfugiés de Conakry mais aussi pour proposer au plus grand nombre une aide au retour dans leurs pays d'origine. Même si je n'ai pas obtenu les résultats définitifs de ce recensement, il ressort des entretiens avec les employés du Bureau guinéen des réfugiés comme des réfugiés eux-mêmes que quelques individus qui n'avaient jamais eu de papiers de réfugiés sont sortis de l'ombre mais que la plupart des Libériens déjà enregistrés ont refusé de se faire compter par peur d'un rapatriement forcé.

Au final, il a été impossible d'avoir une vision statistique globale des réfugiés libériens à Conakry à une date donnée. Les statistiques du HCR tout comme le nombre des ressortissants libériens proposé par l'ambassade sont tout au plus des repères. Pour 2008, si on avance l'hypothèse qu'il y a presque autant de réfugiés libériens sans papiers du HCR que de réfugiés statutaires, tout en prenant en considération que la statistique de l'ambassade libérienne recoupe en partie celle du HCR, on peut proposer une estimation de 7000 réfugiés du Liberia à Conakry en 2008. Ce terrain de thèse n'a pas ambitionné une représentativité des entretiens à effectuer, d'autant plus qu'il s'est heurté au problème de localiser les réfugiés pour les rencontrer.

\section{Choisir des quartiers d'étude? Une méthode inadaptée au terrain}

Les chercheurs géographes travaillant sur des migrants en milieu urbain font parfois des enquêtes par maisons dans des illots de quartier pour rencontrer leurs interlocuteurs ou choisissent des quartiers emblématiques d'immigration (voir par exemple Spire, 2011). La principale raison qui m'a empêchée de recourir à cette méthode est le faible nombre de la population étudiée. Même si on s'appuie sur l'estimation de 7000 réfugiés libériens en 2008, dans une ville où il y aurait environ 1,5 million d'habitants ${ }^{11}$, ils ne compteraient que pour $0,4 \%$ de la population urbaine. En l'absence de camp de réfugiés, les Libériens se sont installés dans les cinq communes de Conakry (Kaloum, Dixinn, Matam, Ratoma et Matoto) dans les différents quartiers de la ville, là où ils ont pu trouver des logements vacants. On ne peut pas identifier de quartiers des réfugiés libériens à première vue. Les Guinéens de la rue rencontrés au hasard des échanges informels ne connaissaient pas de quartiers de réfugiés libériens, ou bien indiquaient le quartier de Bellevue, proche de l'ambassade de Sierra Léone, où avaient été concentrés de nombreux réfugiés, surtout sierra-léonais, à la fin des années 1990. De manière générale, les Libériens étaient d'abord considérés comme des anglophones et souvent confondus avec les Sierra-Léonais.

Comme mentionné ci-dessus, les lieux de résidence des réfugiés ne sont pas enregistrés de façon précise par le HCR, mais la base de données obtenue, malgré ses limites, semble indiquer que les communes de Ratoma et de Matoto sont celles qui accueillent le plus grand nombre de réfugiés ${ }^{12}$. Il s'agit des communes les plus étendues de la ville de Conakry, et les plus peuplées d'après le recensement de 1996. Elles sont parfois appelées «banlieue» par les habitants de la ville alors même qu'elles font partie administrativement de la capitale. Chacune de ces communes est divisée en quartiers. Pour essayer d'identifier des

\footnotetext{
${ }^{11}$ Estimation de l'ONU, 2009. http://data.un.org/CountryProfile.aspx? crName $=\mathrm{G}$ UINEA. Au recensement officiel de 1996, la capitale comptait déjà 1,1 million d'habitants (Bidou, Touré, 2002).

${ }^{12} \mathrm{La}$ commune de résidence a été enregistrée pour seulement $59 \%$ de l'ensemble des réfugiés enregistrés (toutes nationalités confondues - je n'ai pas eu accès au détail individualisé ou par groupes nationaux de ces données) mais 70\% d'entre eux résidaient dans ces deux communes de Ratoma et Matoto début 2008. Il n'y a aucune information, à plus fine échelle, sur les quartiers de résidence.
} 
quartiers de plus forte concentration de réfugiés libériens, je me suis adressée en vain au gouvernorat de Conakry, où l'on m'a à nouveau renvoyée vers le HCR. Formulant l'hypothèse que les chefs de quartier seraient des sources d'information, voire des intermédiaires pour obtenir des entretiens auprès de réfugiés libériens, j’ai mené deux entretiens exploratoires avec des chefs de quartiers à Kaporo (commune de Ratoma) et Simbaya 1 (commune de Matoto). Alors même que les Guinéens sont censés déclarer aux autorités du quartier s'ils hébergent ou ont des locataires étrangers, aucun de mes interlocuteurs ne m'a proposé de rencontrer un réfugié libérien, ni même cité ou montré une maison habitée par un réfugié libérien ${ }^{13}$. Les discours de ces chefs de quartier ont tout de même été très intéressants pour percevoir l'image qu'ils se faisaient des réfugiés en général, et des Libériens en particulier, ainsi que leur représentation des relations entre Guinéens et réfugiés. Malgré certains propos accueillants, il ressort que les réfugiés sont souvent perçus comme un poids, voire une menace pour la société guinéenne, et que le souvenir des attaques frontalières de 2000 reste vivace. Les réfugiés libériens et sierra-léonais, identifiables parce qu'anglophones, ont été considérés comme responsables et complices de ces attaques (McGovern, 2002) et ont été recherchés dans les quartiers pour être arrêtés et rassemblés dans les prisons et les casernes de Conakry. Au-delà de leur discrétion par le nombre, les réfugiés libériens restent discrets dans leurs comportements à Conakry car ils gardent eux aussi un souvenir brûlant de cette chasse aux réfugiés et des violences qui les ont accompagnées. Dans le contexte des troubles qui ont marqué la fin des années de Lansana Conté au pouvoir (manifestations populaires début 2007, mutinerie de l'armée puis de la police mi 2008, puis coup d'État militaire à l'annonce du décès du président

${ }^{13}$ Le chef de quartier de Simbaya 1 a cependant mentionné des cas où il avait été sollicité pour arbitrer des conflits de voisinage entre citoyens guinéens et réfugiés libériens. fin 2008) et face au risque possible d'un retour de xénophobie ciblant les anglophones, considérés comme de possibles mercenaires et fauteurs de troubles, j'ai choisi de ne pas insister auprès des chefs de quartier pour obtenir des entretiens avec des réfugiés libériens, pour ne pas les mettre en danger par rapport à leur environnement social guinéen. Néanmoins, les chefs de quartier m'ont indiqué des églises ou des écoles fréquentées par des anglophones qui ont pu servir de points de départ pour des entretiens, même si la logique du réseau social a davantage pris le pas sur cette méthode d'appui sur des lieux publics ou privés, voire communautaires pour rencontrer des réfugiés libériens ${ }^{14}$.

\section{Passer par l'intermédiaire du HCR ? Le danger de créer la confusion}

Une autre façon d'accéder au terrain, c'est-à-dire aux réfugiés, aurait été de passer par l'intermédiaire du HCR ou des organisations humanitaires engagées auprès des réfugiés. En 2007, lors d'un premier séjour de terrain exploratoire, c'est la méthode que j'ai employée et par laquelle j'ai obtenu mes premiers entretiens avec des réfugiés libériens. Le HCR ou la CroixRouge guinéenne ont ainsi servi d'intermédiaires indirects quand j'ai utilisé leur implantation dans la ville comme un lieu ressource: en me postant quelques jours devant leurs bureaux que les réfugiés libériens fréquentent de façon plus ou moins régulière, j’ai essayé de nouer des contacts. Je n'ai finalement pas persévéré dans cette approche car de nombreux réfugiés ont pensé, probablement à cause de ma couleur

\footnotetext{
${ }^{14} \mathrm{Au}$ final, j'ai en effet davantage mené des entretiens dans des lieux fréquentés par des réfugiés libériens (églises, écoles, centre informel d'hébergement de passage) après y avoir été introduite par des informateurs libériens. Il s'agit surtout d'un appui a posteriori sur ces lieux communautaires, tandis qu'il est plutôt antérieur à tout contact dans le travail d'Olga Bronnikova pour observer les réseaux sociaux des migrants russes à Paris et favoriser les entretiens (Bronnikova, 2009).
} 
de peau, que j'étais une nouvelle employée et ont souhaité à tout prix me parler. Même après m'être présentée à plusieurs personnes et avoir expliqué ma recherche, l'incompréhension demeurait, notamment parce que je disais ne vouloir rencontrer que les réfugiés du Liberia. Un Sierra-Léonais m'a alors dit "We are all refugees, we are all suffering!». On retrouve avec ce terme de souffrance un autre aspect sensible de ce terrain, mais il me semble aussi important de souligner que le lieu et le cadre jouent un rôle important, comme j'ai pu le constater au fur et à mesure des enquêtes : ils font varier le contenu des témoignages recueillis. Mener des entretiens en pleine rue, avec de nombreuses oreilles proches et si près d'institutions où les personnes rencontrées venaient en position de demandeur n'était pas toujours propice au recueil de l'information. Seuls quelques-uns de ces contacts se sont transformés en entretiens mobilisés pour la thèse, le plus souvent parce que j'ai rencontré mes interlocuteurs dans un contexte moins sensible, au sens où il était moins chargé d'enjeux symboliques et où mes questions sur un projet migratoire ne prenaient pas la même résonnance à deux pas du HCR que dans une arrière-cour privée.

De manière plus directe, j’ai obtenu d'une employée du HCR les coordonnées téléphoniques de quelques réfugiés libériens. J'ai ainsi pu rencontrer L., à laquelle j'ai donné rendez-vous devant le HCR, puis qui a accepté de m'emmener chez elle pour un entretien. Changer de lieu a contribué à rendre sa parole plus libre, par rapport à sa perception du HCR, mais aussi pour raconter sa vie, dans un environnement plus intime. Cependant, la parole de L. est restée comme corsetée car elle savait que je la rencontrais par l'entremise du HCR. Elle doutait probablement de mon objectif, même après avoir vu ma carte d'étudiante et entendu ma thématique de recherche. L. avait d'ailleurs été sélectionnée par l'employée du HCR comme étant une «bonne » réfugiée, avec laquelle je ne serais pas en danger, et qui ne critiquerait pas trop l'institution. Ce filtre du HCR était problématique pour mener ma recherche, d'autant plus que l'agence me proposait surtout de rencontrer les réfugiés avec lesquels elle avait l'habitude d'échanger et qui connaissaient bien le «vocabulaire » du HCR. C'est notamment le cas du «comité des réfugiés libériens », c'est-à-dire les représentants élus des réfugiés libériens enregistrés au HCR. Il a été intéressant de rencontrer ces personnes charismatiques et loquaces, dont le discours était particulièrement "politisé » et communautaire, mais j'ai finalement préféré le faire sans passer par le HCR. Utiliser une organisation humanitaire ayant un pouvoir sur les réfugiés créait un biais trop important et problématique: les réfugiés ne croient jamais tout à fait que le chercheur n'a aucun lien avec l'organisation qui l'a recommandé (Baujard, 2008, pp. 39-42). Ils en viennent à adopter des discours formatés, destinés à l'organisation, ou du moins à veiller à leurs propos car ils redoutent qu'ils soient rapportés, et qu'on puisse y constater des écarts avec ce qu'ils auraient pu déclarer par ailleurs.

Ayant envisagé plusieurs façons d'aborder mon terrain et d'accéder aux réfugiés, et ayant même testé certaines approches, comme je viens de l'évoquer, j’ai finalement choisi de m'appuyer sur les réseaux sociaux pour rencontrer les réfugiés. Même si cette méthode présente d'autres biais, que j'essaierai d'évoquer ici, elle m'a permis de mener un grand nombre d'entretiens, à travers toute la ville et avec des individus aux parcours très différents dont j’ai essayé de gagner la confiance.

\section{Rencontrer les réfugiés}

Pour rencontrer les réfugiés libériens à Conakry, je me suis appuyée sur les réseaux sociaux de mes informateurs, ce que je vais décrire ici. C'est en recourant à cette méthode que j'ai pu contourner la difficulté du faible nombre de réfugiés et de leur 
dispersion dans la ville, sans recourir à des intermédiaires institutionnels. Le caractère souple de cette approche présente des avantages et des inconvénients qu'il s'agit de présenter, tout en essayant de montrer qu'elle a permis d'ouvrir des thèmes de réflexion en lien avec mon questionnement central sur les projets migratoires des réfugiés.

\section{Recourir au bouche à oreille ? Les avantages de la méthode " boule de neige"}

La méthode de la «boule de neige » est souvent décrite dans les manuels de sociologie comme une méthode empirique utile dans les situations où le groupe étudié est en petit nombre car il présente des caractéristiques précises et restreintes, et où les personnes à interviewer sont difficiles à localiser, notamment quand il n'existe pas de liste exhaustive de cette population (Mercklé, 2004). Elle s'appuie sur le premier entretien qui en génère d'autres car la personne rencontrée introduit le chercheur à d'autres personnes ayant les mêmes caractéristiques. L'image de la boule de neige indique qu'il est ensuite facile de multiplier les entretiens. En anglais, on parle aussi de snowball sampling ou encore de referral sampling car une personne renvoie vers plusieurs autres mais aussi parce qu'elle sert de référence au chercheur. Le terme de référence est ici double. D'une part, la personne initiale présente des caractéristiques d'intérêt pour le chercheur Jean-Pierre Olivier de Sardan propose même de parler de « cas extrême de groupe social témoin restreint à un individu » (Olivier de Sardan, 1995). D'autre part, elle lui permet de gagner plus facilement la confiance d'autres informateurs potentiels en recommandant le chercheur auprès de son réseau social, ce qui sera discuté plus loin. Pour les études sur les réfugiés urbains, cette méthode est régulièrement évoquée et discutée par les chercheurs (Jacobsen, Landau, 2003; American University of Cairo, 2003).

Figure $\mathrm{n}^{\circ} 2$ : Les réfugiés rencontrés par méthode « boule de neige » à partir d'un premier contact.

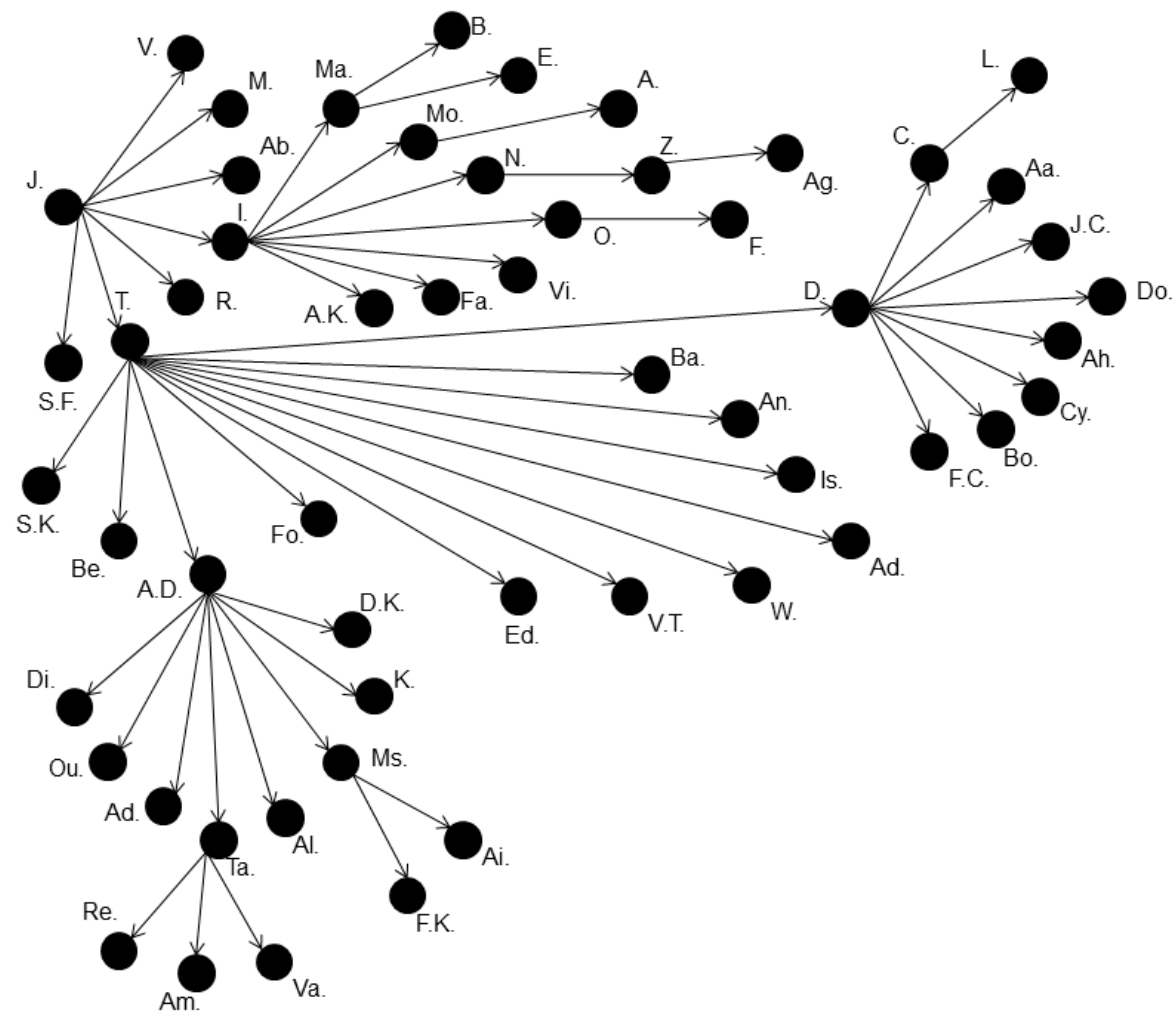

Source : H. Simon-Lorière, 2007. 
Dès mon premier terrain en 2007, j’ai essayé de faire fonctionner le bouche à oreille pour rencontrer des réfugiés. J'ai parlé de mon projet de recherche à plusieurs personnes dans le but d'accéder au terrain non pas par le biais d'une institution, mais par l'entremise d'individus. C'est ainsi que j'ai rencontré Madame B., une restauratrice de rue guinéenne qui avait vécu en Sierra Léone, qui attirait dans son maquis une clientèle de réfugiés parce qu'elle proposait des plats léonais et libériens et que son menu était aussi en anglais. Dans son restaurant, j'ai fait la connaissance de J., un réfugié libérien, qui m'a ensuite ouvert son réseau de relations sociales. Grâce à J., puis à d'autres réfugiés qui m'ont à leur tour présenté leurs relations, $j$ 'ai rencontré une cinquantaine de personnes, comme l'illustre la figure $n^{\circ} 2$.

Pour accéder au terrain et multiplier les contacts et les possibilités d'entretiens, je me suis appuyée sur plusieurs informateurs : à la fois ceux rencontrés grâce aux relations de J., mais aussi sur d'autres informateurs rencontrés grâce à des contacts guinéens ${ }^{15}$, et sur des informateurs qui m'avaient euxmêmes contactée. En effet, les réfugiés déjà interviewés auxquels j’avais laissé systématiquement mon numéro de téléphone l'ont donné à d'autres : une partie des entretiens suivants a alors pu se faire sans intermédiaire direct. Je me suis efforcée de mener les entretiens au domicile des réfugiés, ou bien sur leur lieu de travail, ce qui a été possible dans $60 \%$ des cas, même si des lieux publics ou bien la maison d'un informateur ont été le cadre du reste des entretiens. Rencontrer les individus chez eux donnait un cadre plus familier pour la conversation tout en me permettant d'observer concrètement leurs conditions de vie. Après un contact par téléphone, un rendez-vous donné dans un quartier, sur un axe principal de la ville, permettait de trouver la personne et l'entretien avait ensuite lieu chez elle, alors que dans les cas où le réseau personnel d'un individu permettait la rencontre, la localisation des réfugiés était facilitée par l'informateur. Par exemple, A.D., un informateur que l'on retrouve sur la figure $\mathrm{n}^{\circ} 2$ (J. a permis de rencontrer $\mathrm{T}$. qui a permis de rencontrer A.D.), m'a donné rendez-vous à Lambanyi (commune de Ratoma), quartier périphérique de Conakry où il réside, et emmenée chez des compatriotes qui vivaient dans la même zone. La méthode «boule de neige » a ainsi facilité la localisation des réfugiés, en contournant la quasi absence de données, et a apporté une matérialisation de la dispersion des réfugiés à travers la ville. C'est ce qu'illustre la figure $n^{\circ} 3$ qui montre les quartiers de résidence des Libériens rencontrés. Le plus grand nombre d'entretiens a été effectué dans certains quartiers périphériques, comme Simbaya Gare (Ratoma) ou Tombolia (Matoto). Il s'agit là d'un effet de l'application de la méthode «boule de neige » mais on peut avancer l'hypothèse que les réfugiés se seraient installés de façon privilégiée dans certains quartiers. Il faut ensuite chercher des explications de ce phénomène du côté d'une part, de l'expansion de la ville et du marché du logement à Conakry au moment de leur arrivée, ou d'autre part, de regroupements par affinités ethniques.

\footnotetext{
${ }^{15}$ Une démarche similaire de composition progressive de réseaux d'informateurs est décrite par Marjolaine Paris, (Paris, 2009).
} 
Figure $\mathrm{n}^{\circ} 3$ : Les quartiers de résidence des réfugiés libériens rencontrés à Conakry.

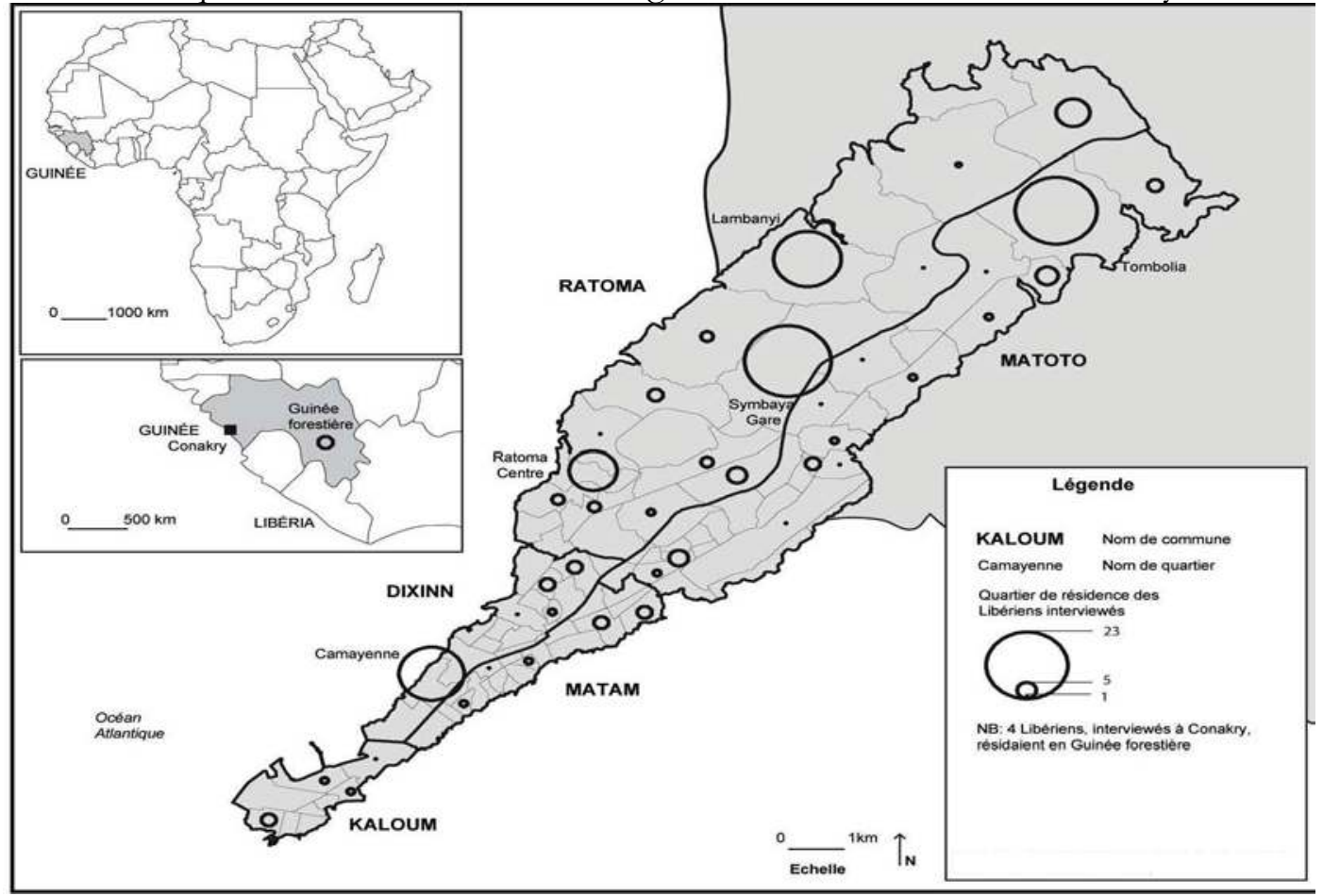

Source : Fond de carte Mapinfo (remerciement à D. Bazzo). Réalisation : H. Simon-Lorière, 2012.

Au total, 200 entretiens ont été réalisés à Conakry, principalement grâce à cette méthode. Le capital social des individus est devenu un élément de réflexion à part entière pour mieux appréhender les différentes tactiques des Libériens pour leur survie individuelle et de groupe d'une part et pour leurs projets migratoires d'autre part. La notion de capital social découle de cette observation des relations sociales des individus. Elle implique que ces relations sont des ressources et lui apportent du pouvoir à la fois pour mener des actions collectives, mais aussi pour fonder «des stratégies individuelles organisées autour de [leur] utilisation » comme l'ont montré les travaux de James Coleman (Mercklé, 2004, p. 54). Dès la mise en œuvre de la méthode d'enquête, de cette "politique du terrain» décrite ici, il m'a semblé important et intéressant de percevoir les réfugiés comme des acteurs, développant peut-être moins des stratégies que des tactiques, au sens de de Certeau (1980). Car il ne s'agit pas de nier l'étroitesse de leur marge de manœuvre, tant pour essayer d'améliorer leurs conditions de vie à Conakry, dans un pays pauvre, que l'indice de développement humain place parmi les vingt derniers pays du monde dans le classement de l'ONU, que pour essayer de partir à l'étranger vers des pays développés aux politiques migratoires de plus en plus restrictives. Les réseaux ont par exemple été un moyen d'interroger la «solidarité» souvent invoquée pour décrire les conditions de vie des réfugiés. Dans sa thèse, Roos Willems a analysé la richesse des réseaux des réfugiés à Dar Es Salam (2003), en s'appuyant sur les notions de «liens forts» et de « liens faibles » : selon elle, les individus aux "liens forts", c'est-à-dire aux réseaux denses et homogènes, reçoivent plus d'aide en cas d'urgence que ceux aux réseaux larges et divers. J'ai pu essayer de vérifier cette idée à Conakry, notamment en interrogeant particulièrement les réfugiés sur les aides financières (remittances) que certains d'entre eux recevaient de l'étranger. En plus de cette dimension sociale, le recours aux réseaux sociaux invitait aussi à réfléchir à la notion, plus politique, de «communauté réfugiée libérienne», employée notamment par le HCR quand il cherche à dégager des interlocuteurs et des relais, comme ce 
«comité des réfugiés " évoqué plus haut, pour expliquer les programmes qu'il met en place pour les réfugiés ${ }^{16}$. Dans les entretiens mais aussi en observant des réunions de réfugiés, j’ai pu apercevoir des éléments d'unité du groupe ainsi que des lignes de fractures, particulièrement révélées dans les relations avec le HCR. Porter la parole des réfugiés au HCR est un enjeu politique dont j'ai eu plusieurs fois l'occasion de parler sur le terrain avec mes interlocuteurs, ce qui a été observé ailleurs par d'autres chercheurs (Baujard, 2008). Sur le terrain, j’ai pu constater la place politique et sociale de certains individus charismatiques dont le capital social était important et qui sont parfois devenus des informateurs très utiles. D'autres individus avaient fait le choix d'être plus autonomes par rapport au HCR et au groupe des réfugiés et privilégiaient des liens divers, plus ouverts aux Guinéens, notamment dans leur voisinage immédiat ${ }^{17}$.

\section{S'appuyer sur les réseaux sociaux ? Les limites de la méthode}

La première limite de la méthode «boule de neige » est celle du manque de représentativité (Jacobsen, Landau, 2003). Sans connaitre le nombre total de réfugiés libériens à Conakry, il est difficile d'envisager un échantillon représentatif, mais l'utilisation du réseau social d'un individu entraîne le risque de rencontrer des individus aux

\footnotetext{
${ }^{16}$ Par programmes, je fais ici tout particulièrement allusion à ce que le HCR appelle les «solutions durables »: le rapatriement librement consenti (le retour au pays), l'intégration locale (le fait de rester dans le pays d'asile) et la réinstallation (l'installation dans un pays développé qui propose des quotas d'accueil pour les réfugiés) évoqués en introduction pour discuter des perspectives migratoires des réfugiés.

${ }^{17} \mathrm{Ceci}$ ne veut pas dire que les réfugiés qui ont paru les plus impliqués dans ce que j'appellerai le «lobbying » auprès du HCR n'avaient pas développé de relations sociales avec des Guinéens. Cependant, il m'a fallu davantage de temps et plusieurs entrevues avec ces personnes - l'itération, dont parle Jean-Pierre Olivier de Sardan (Olivier de Sardan, 1995) pour observer ces relations que mes interlocuteurs ne mentionnaient pas dans nos échanges.
}

caractéristiques très semblables. Sur les 200 entretiens par exemple, une personne sur cinq a connu un exil par la Sierra Leone, et de nombreux réfugiés se connaissaient en effet pour avoir vécu dans le même camp, «Waterloo camp », aux portes de la capitale, Freetown. Il s'agit là d'un biais, qui a même parfois donné lieu à des récits et des parcours d'exil stéréotypés, mais qui a aussi mis en relief la force de ce réseau d'expérience, à partir des épreuves partagées dans ce camp de réfugiés qui a été détruit en 1997 par l'avancée des rebelles de la guerre civile sierra léonaise. Même si les profils des personnes rencontrées sont relativement variés (en termes de parcours migratoires, de situation familiale ou encore d'activités économiques), la principale faiblesse de mon travail de terrain est qu'il a finalement touché une minorité de réfugiés sans papiers, la majorité étant des réfugiés statutaires. L'approche par les réseaux sociaux n'a pas réellement permis de rencontrer des Libériens ayant fui la guerre mais ayant refusé de se considérer comme des réfugiés.

Un autre biais important dans l'application de cette méthode a été le poids des intermédiaires. Dans plusieurs situations, il m'a été impossible de m'en défaire. Quelques informateurs ont en effet essayé de contrôler le processus des entretiens. Avec l'un d'entre eux, il a fallu que j'insiste pour être laissée seule avec les interlocuteurs qu'il me présentait. J'ai ensuite contourné le problème en prenant le numéro de téléphone des réfugiés rencontrés pour ménager la possibilité d'autres entrevues et compléter une première conversation trop contrainte par les éléments extérieurs. Dans le cas d'A., une réfugiée initialement rencontrée alors qu'elle travaillait dans la petite boulangerie de l'une de mes informatrices, les premières informations relevées ont été peu nombreuses parce qu'elle était en plein travail (même si les fournées de gâteaux laissaient le temps à la discussion) mais aussi parce qu'elle n'était pas tout à fait libre de répondre aux questions les plus personnelles au milieu des 
autres travailleurs. Un autre informateur avait convoqué une foule de personnes dans sa cour (près de 200 personnes, en majorité illettrées), créant ainsi des attentes surdimensionnées par rapport à l'objectif de mes entretiens. À trois reprises, je me suis rendue chez lui et j'ai mené des entretiens avec quelques-uns des réfugiés présents mais j'ai ensuite décidé de ne pas continuer ainsi. Comment être sûre que ma présence n'était pas présentée comme autre chose qu'une enquête universitaire? L'informateur pouvait avoir fait courir la rumeur que des visas seraient à la clé des entretiens et pouvait les avoir monnayés...

Enfin, la méthode entraîne un certain rythme sur le terrain : au début, le processus est lent à se mettre en place, puis on atteint un seuil, où le bouche à oreille fonctionne à plein et les possibilités d'entretiens se multiplient, jusqu'à atteindre un autre seuil de saturation pour un enquêteur seul. Après plusieurs semaines, de nouveaux réfugiés m'appelaient régulièrement pour demander un entretien et je n'ai pas été en mesure de tous les rencontrer. À l'échelle des journées, ce seuil de saturation était également présent car j’ai parfois enchainé 6 à 8 entretiens. Cela a créé de la frustration et des entretiens parfois moins aboutis à cause d'une baisse du degré d'attention aux détails des récits ou d'un manque de réactivité dans les questions. On retrouve là encore l'aspect sensible du contenu des entretiens: comment appréhender l'interlocuteur et l'entretien suivant quand on vient d'entendre un récit d'assassinat, de viol, de maladies, de morts et de souffrances? La densité dramatique mais aussi la richesse de l'entretien quant à mes thèmes de questionnement dépendaient néanmoins de chaque individu et si la qualité du premier contact noué était moins bonne, elle a pu être retravaillée sur le temps long par d'autres rendez-vous pour quelques cas. Surtout, la notion de saturation prend son sens d'un point de vue scientifique car les entretiens supplémentaires apportaient de moins en moins d'informations nouvelles et tendaient de plus en plus à entrer dans les catégories d'analyse qui avaient émergé, pour les parcours migratoires, les conditions de vie à Conakry et surtout pour les interrogations autour du projet migratoire.

\section{Faire parler les réfugiés ? Les risques de l'incompréhension et du mensonge}

Un dernier élément d'analyse quant au bouche à oreille, qui peut être interprété à la fois comme un avantage et comme un inconvénient de la méthode, concerne la confiance et la transparence entre le chercheur, l'informateur et l'enquêté. Les entretiens que j'ai sollicités ont en effet créé beaucoup d'attentes parmi les réfugiés, en lien avec leurs projets migratoires, au risque de susciter des malentendus et des récits mensongers.

À chaque nouvelle rencontre, la présentation de soi et du travail de thèse en cours était primordiale. Cette présentation était souvent faite par l'informateur, mais je prenais aussi le temps de me présenter et de parler de mon projet de recherche. Montrer une carte universitaire a été utile pour dissiper certains doutes. Cette démarche s'est mise au point sur le terrain, après quelques discussions avec des réfugiés et des travailleurs du HCR. Les uns comme les autres insistaient sur le risque que les Libériens pensent que les entretiens s'inscrivent dans un programme de réinstallation et qu'ils ouvraient des possibilités migratoires, ou bien tout simplement une aide matérielle. Le fait que je sois une femme blanche pouvait faciliter les malentendus (Aggoun, 2009; American University of Cairo, 2003). Même quand quelques mots avaient été dits devant un groupe pour me présenter, ou que les réfugiés avaient déjà entendu parler de moi par leurs relations, je répétai à nouveau au début de chaque entretien mon statut de chercheuse. À chaque personne, je précisais que je ne travaillais pas avec le HCR, une ONG ou une ambassade, et que je n'allais apporter aucune aide concrète. Malgré ces précautions oratoires, une jeune femme m'a 
un jour demandé à la fin d'une interview si j'étais australienne : elle avait entendu parler d'une mission du gouvernement australien en vue d'accueillir des réfugiés, et pensait que je cachais ma réelle identité... Sur le terrain, cette préoccupation que l'activité de recherche ne soit pas déformée ou détournée par certains réfugiés a été centrale. Si l'information que je cherchai à mener des entretiens auprès de réfugiés libériens s'est répandue, il s'agissait aussi de s'assurer que l'objectif des entretiens soit clair pour chaque individu interviewé.

Instaurer la confiance avec les réfugiés pour faciliter le dialogue et recueillir des récits de vie était un objectif qui n'a pas été atteint avec chacune des 200 personnes enquêtées. $\mathrm{Au}$ final, des échanges approfondis et répétés n'ont été possibles qu'avec une vingtaine de personnes et les récits de vie récoltés sont souvent partiels et lacunaires. Néanmoins, le fait d'être introduite à un nouveau contact par une personne qui lui était familière a pu faciliter la confiance. Il s'agit là d'une relation triangulaire qui implique une volonté de transparence et d'échange de la part du chercheur, de l'informateur et de l'enquêté. Le plus souvent, j'ai essayé de faire des entretiens en tête à tête, pour préserver une certaine intimité. Dans quelques cas, les entretiens ont été menés devant un groupe : chacun a pu constater que le même type de questions était posé et qu'il n'y avait pas de secrets. Tous les entretiens ont eu lieu en anglais libérien, sans traducteur (sauf avec deux vieilles femmes qui ne parlaient que leur dialecte). Face à des personnes illettrées, j'étais amenée à répéter, voire reformuler mes questions. Au fur et à mesure des rencontres, ma compréhension de l'anglais libérien s'est améliorée. Ma connaissance du conflit libérien, et surtout, après un séjour à Monrovia, ma connaissance de la capitale libérienne, ont été des éléments importants de connivence, même si dans certains cas faire celle qui ne connaissait pas permettait de recueillir des informations plus précises et riches. Dans la perspective d'analyser finement les témoignages des réfugiés, j'ai choisi d'enregistrer les entretiens. La présence d'un dictaphone, qui n'a jamais été un obstacle pour les Libériens, m'a libérée d'une prise de note exhaustive et a facilité les échanges de regards. Même si une trame de thèmes et de questions était suivie pour chaque interview, la méthode d'enquête a été adaptée aux interlocuteurs, à leur profil, notamment leur niveau scolaire, à leur degré d'ouverture et à leurs attentes. Par conséquent, la durée des entretiens varie beaucoup d'un individu à l'autre. Certains réfugiés ont été plus curieux et m'ont posé davantage de questions personnelles, comme pour maintenir un niveau d'information égal. D'autres ont cherché à savoir le bénéfice qu'ils tireraient d'un entretien. Comme l'écrivent plusieurs chercheurs ayant travaillé avec des réfugiés, la méfiance et la suspicion sont des éléments récurrents dans les enquêtes, mais ces sentiments se retrouvent autant du côté des enquêtés que celui du chercheur (Bouillon, Fresia, Tallio, 2006). En travaillant sur les enquêtes, je me suis posée la question du mensonge dans quelques cas où les récits devenaient incohérents entre deux rendez-vous, voire dans un même entretien. Certains éléments qui semblaient mensongers pouvaient correspondre à une façon de taire des expériences personnelles et douloureuses. Mais le mensonge pouvait aussi concerner des expériences douloureuses, surexposées ou réappropriées dans une tactique consciente de l'importance de ce type de critère dans les programmes de réinstallation. Il a fallu accepter ce risque et prendre les témoignages comme ils venaient, même si la possibilité d'éléments inventés ou au contraire occultés est ensuite à prendre en compte dans l'analyse des récits (American University of Cairo, 2003). Le conflit du Liberia lui-même n'étant pas au cœur de l'étude, j'ai parfois fait le choix d'abréger les questions concernant l'expérience de la guerre, dont quelques individus ont d'ailleurs tout à fait refusé de parler, mais je souhaitais tout de même récolter des informations sur les modalités de la fuite hors du Liberia et de 
l'exil. En effet, un des présupposés de mon travail sur les projets migratoires des réfugiés est qu'ils prennent sens au regard de leurs expériences individuelles et familiales passées.

\section{Conclusion}

Les réseaux sociaux se sont imposés à Conakry comme un outil de travail pour accéder aux réfugiés libériens. La méthode de la «boule de neige », même si elle présentait d'autres biais, m'a permis de m'affranchir du HCR comme informateur, et d'être plus proche des réfugiés, à la manière des anthropologues. Cette méthode d'enquête a été une adaptation à ce terrain. Néanmoins, l'adjectif sensible, pour lequel je donnai une précision sémantique en introduction à cet article en citant un ouvrage consacré aux terrains sensibles (Bouillon, Fresia, Tallio, 2006), correspond finalement moins aux conditions d'enquête à Conakry et à la méthode, qu'au contenu des entretiens, porteurs d'expériences de souffrances et de violences et surtout aux enjeux que mon questionnement sur les projets migratoires soulevait. Même si le «protocole d'enquête" déployé à Conakry n'a pas été «trop canonique », le recours aux réseaux sociaux a surtout permis d'aborder de façon détournée la question des projets migratoires. Les réseaux sociaux ont tout autant joué un rôle méthodologique, pour localiser et rencontrer les réfugiés, qu'heuristique, pour appréhender leurs trajectoires, leurs conditions de vie présentes et leurs perspectives migratoires. Selon l'échelle d'observation des réseaux, celle de la ville de Conakry et celle plus vaste du monde, l'usage et l'analyse de ces réseaux a joué un rôle différent dans ma recherche. En interrogeant les réseaux sociaux des réfugiés libériens, j'ai pu reconstituer des liens transnationaux créés à cause de la dispersion provoquée par la guerre civile libérienne. $W e$ all scattered est une expression récurrente dans les entretiens recueillis, de même que le fait que les réfugiés ne sachent pas (ou déclarent ne pas savoir) où sont certains membres de leur famille. Percevoir si les réfugiés de Conakry avaient des contacts avec leurs compatriotes, restés au pays, exilés dans d'autres pays d'Afrique de l'Ouest ou dans des pays développés a été assez difficile. Rares sont les réfugiés qui ont tout de suite annoncé être en contact avec des membres de leur famille, que ce soit ailleurs en Guinée, en Afrique ou en Occident. Pour les personnes rencontrées à plusieurs reprises, l'information est intervenue avec le temps et l'établissement d'une relation de confiance. Le projet migratoire est un sujet sensible car les réfugiés ne souhaitent pas forcément en parler directement et peuvent préférer garder secrètes leurs tactiques en vue d'un éventuel départ de Conakry, quelle qu'en soit la destination. Un réfugié m'a finalement confié qu'il avait des enfants dans le camp de réfugiés de Buduburam au Ghana laissant entr'apercevoir une tactique de «plurilocalisation» (Monsutti, 2004, p. 63) pour maximiser les chances familiales d'accéder à une réinstallation dans un pays développé. Ces tactiques, qui vont à l'encontre des principes du HCR et des règles des pays d'accueil, de même que les déplacements entre pays d'asile pour rechercher de meilleures conditions de vie et de plus grandes opportunités de départ sont plutôt cachées par les réfugiés. Dans ma recherche, la reconstitution des réseaux a permis de ne pas aborder de front la question sensible du projet migratoire, mais plutôt de glaner au fur et à mesure des informations à ce sujet.

Hélène Simon-Lorière Doctorante en Géographie Migrinter - UMR 7301 CNRS / Université de Poitiers hsimonloriere@gmail.com 


\section{Bibliographie}

Aggoun, Atmane (dir.) (2009) Enquêter auprès des migrants, Le chercheur et son terrain, L'Harmattan, Paris, 164 p.

Agier, Michel (2008) Gérer les indésirables. Des camps de réfugiés au gouvernement humanitaire, Paris, Flammarion, 350 p.

American University of Cairo, Forced Migration and Refugee Studies Programme, (2003) Workshop report: Research on Refugees in Urban settings: Methods and Ethics, 13 p. [en ligne, réf. du 20 mai 2007] URL: <http://www.aucegypt.edu/fmrs/outreach/wor kshops/Urbanworkshop.pdf>

Andrews Gale, Lacey (2008) The invisible refugee camp : durable solutions for Boreah 'residuals' in Guinea, Journal of Refugee Studies, vol. 21, N4, pp. 537-552.

Baujard, Julie (2008) Identité "réfugiê", identité transversale. Les réfugiés à Delbi au sein des dynamiques institutionnelles, communautaires et associatives, Université d'Aix-Marseille 1, 502 p. [en ligne, réf. du 13 avril 2012] URL: $<$ http://tel.archives-

ouvertes.fr/docs/00/35/68/95/PDF/these. pdf $>$

Th. Doct : Anthropo. : Marseille : 2008.

Bidou, Jean Etienne; Toure, Julien Gbéré (2002) La population de la Guinée dynamiques spatiales, Les Cahiers d'Outre-Mer, $\mathrm{N}^{\circ} 217$, [en ligne, réf. du 13 avril 2012] URL :

$<$ http://com.revues.org/index1049.html $>$

Black, Richard (1999) Les réfugiés dégradent-ils l'environnement ? Exemples au Sénégal et en Guinée, in Lassailly-Jacob, Véronique; Marchal, Jean-Yves; Quesnel, André (dir.), Déplacés et réfugiés: la mobilité sous contrainte, Paris, IRD, pp. 383-408.

Bouillon, Florence ; Fresia, Marion; Tallio, Virginie (dir) (2006) Terrains sensibles: expériences actuelles de l'anthropologie, Paris, Édition de l'EHESS, 208 p.
Bronnikova, Olga (2009) Observer les réseaux sociaux chez les migrants russes en France in Aggoune Atmane (dir.), Enquêter auprès des migrants, le chercheur et son terrain, Paris, L'Harmattan, pp. 93-119.

Calbérac, Yann (2010) Terrains de géographes, géographes de terrain: communauté et imaginaire disciplinaires au miroir des pratiques de terrain des géographes français du XXe siècle, Université Lumière Lyon 2, 396 p. [en ligne, réf. du 13 avril 2012] URL: <http://tel.archivesouvertes.fr/docs/00/55/14/81/PDF/these cal berac.pdf $>$

Th. Doct : Géogr. : Lyon : 2010.

Crisp, Jeff (1999) Who has counted the refugees? UNHCR and the politics of numbers, New Issues in Refugee Research, Working Paper $n^{\circ} 12$, [en ligne, réf. du 13 avril 2012] URL : $<$ http://www.reliefweb.int/rw/lib.nsf/db900SI D/LGEL-5RRDWP?OpenDocument>

De Certeau, Michel (1980) L'invention du quotidien. Arts de faire, Paris, Union générale d'éditions, $374 \mathrm{p}$.

Ferry, Fleur (2004) La présence des réfugiés fuyant la guerre en Guinée forestière, à l'origine de mutations du réseau et des espaces urbains, in Grünewald, François ; Levron, Eric (dir.), Villes en guerre et guerres en ville: pratiques humanitaires en questions, Paris, Karthala, pp. 221-280 (Pratiques humanitaires).

Jacobsen, Karen (2006) Refugees and Asylum Seekers in Urban Areas: A Livelihoods Perspective, Editorial Introduction, Journal of Refugee Studies, vol. 19, $\mathrm{n}^{\circ} 3$, , pp. 273-286 (Special Issue Urban Refugees).

Jacobsen, Karen; Landau, Loren (2003) Researching refugees: some methodological and ethical considerations in social science and forced migration, Forced Migration Working Paper, $\mathrm{n}^{\circ} 2$, University of the Witwatersrand, 28 p. [en ligne, réf. du 13 avril 2012] URL : $<$ http://repository.forcedmigration.org/show metadata.jsp?pid $=$ fmo:5803> 
McGovern, Mike (2002) Conflit régional et rhétorique de la contre-insurrection. Guinéens et réfugiés en septembre 2000, Politique Africaine, n 88 , pp. 84-102.

Mercklé, Pierre (2004) Sociologie des réseaux sociaux, Paris, La Découverte, $121 \mathrm{p}$.

Monsutti, Alessandro (2004) Guerres et migration. Réseaux sociaux et stratégies économiques des Hazaras d'Afghanistan, Paris, Editions de la Maison des Sciences de l'Homme, 364 p.

Olivier de Sardan, Jean-Pierre (1995) La politique du terrain, Enquête, n 1 , pp.71-109 [en ligne, réf. du 13 avril 2012] URL: $<$ http://enquete.revues.org/document263.html $>$

Paris, Marjolaine (2009) Enquêtes auprès d'élites économiques immigrées en France et au Nigeria : gérer les frontières ethniques, de classes et de statuts, in Aggoune Atmane (dir.), Enquêter auprès des migrants, Le chercheur et son terrain, Paris, L'Harmattan, pp. 31-64.
Spire, Amandine (2011) L'étranger et la ville en Afrique de l'Onest, Lomé au regard d'Accra, Paris, Karthala, 378 p.

Van Damme, Wim (1999) Les réfugiés du Liberia et de Sierra Leone en Guinée forestière (1990-1996), in Lassailly-Jacob, Véronique; Marchal, Jean-Yves; Quesnel, André (dir.), Déplacés et réfugiés: la mobilité sous contrainte, Paris, IRD, pp. 343-381.

Willems, Roos (2003) Embedding the refugee experience: forced migration and social networks in Dar Es Salam, Tanzania, Université de Floride, 429 p. [en ligne, réf. du 13 avril 2012]

URL :

$<\underline{\text { http://etd.fcla.edu/UF/UFE0002281/willems }}$ r.pdf $>$

Th. Doct : Anthropo. : Floride : 2003. 